\title{
Positron Annihilation Study of Defects Induced by Various Cutting Methods in Stainless Steel Grade 304
}

\author{
P. Horodek · J. Dryzek $\cdot$ M. Wróbel
}

Received: 12 July 2011 / Accepted: 9 November 2011 / Published online: 22 November 2011

(C) The Author(s) 2011. This article is published with open access at Springerlink.com

\begin{abstract}
We report in this article the comparison of the subsurface zones (SZs) in austenitic stainless steel 304 samples created by three cutting techniques, i.e., the laser cutting (LC), abrasive water jet (AWJ), and traditional milling cutting (MC). The crystal lattice defects distribution in this zone were investigated using the positron annihilation method. It was shown that the MC creates the great number mainly edge dislocations decorated by vacancies. Their concentration decreases with the increase of the depth from the cut surface. The total depth of the SZ is extended up to $150 \mu \mathrm{m}$ from the cut surface. Similar dependency was observed for the AWJ machining but the total depth is much lower, i.e., $40 \mu \mathrm{m}$. The laser cut machining generates the zone with the lowest concentration of defects which does not vary with the depth. However, LC affects the sample up to the depth of about 500 $\mu \mathrm{m}$. The degree of the surface roughness is similar for laser cut and AWJ, but for the MC it is five times smaller.
\end{abstract}

P. Horodek $(\bowtie) \cdot$ J. Dryzek

Institute of Nuclear Physics PAN, Ul. Radzikowskiego 152,

31-342 Kraków, Poland

e-mail: Pawel.Horodek@ifj.edu.pl

J. Dryzek

e-mail: Jerzy.Dryzek@ifj.edu.pl

J. Dryzek

Institute of Physics, Opole University,

Ul. Oleska 48, 45-052 Opole, Poland

M. Wróbel

AGH University of Science and Technology,

Ul. Mickiewicza 30, 30-059 Kraków, Poland

e-mail: mwrobel@agh.edu.pl
Keywords Stainless steel - Surface layer - Defect profile · Machining - Laser cutting - Abrasive water jet cutting · Positron annihilation spectroscopy

\section{Introduction}

Important features of a surface formed as a result of material removal in the machining process are its texture and integrity. Machining causes also alternations in the layer or zone below the cut surface, for instance, subsurface deformation or hardening. The presence of the subsurface zone (SZ), physical properties of which differ from those in the interior of a material [1] may influence the performance of the surface in service. For inspection of such alternations profilometry, microscopy, hardness testing, SEM, TEM [2] X-ray diffraction can be used [3, 4]. Positron annihilation spectroscopy is seldom used for this purpose; however; it is a widely used technique for detecting of the defects at the atomic scale [5-8]. The great sensitivity of positron techniques to the open volume defects, like vacancies, their clusters, dislocations, and grain boundaries should be helpful in the studies of SZ induced by machining.

Profiles of defects in the SZ have already been studied using positron techniques. Hansen and Petersen [9] reported the total depth of the SZ in pure molybdenum after cutting with the diamond saw equal to $150 \mu \mathrm{m}$. The small vacancy clusters which contain a few vacancies on the depth $20 \mu \mathrm{m}$ and a small amount of monovacancies between 20 and $40 \mu \mathrm{m}$ below the surface were observed. In pure well-annealed $\mathrm{Cu}$ after cutting using a lathe tool, the range of defective zone extends up to $400 \mu \mathrm{m}$, and the characteristic exponential decay of the measured positron annihilation characteristic with the depth was detected 
[10]. Cutting of $\mathrm{Cd}_{1}-{ }_{x} \mathrm{Zn}_{x}$ Te wafers by means of a singlewire saw caused damage of the material below the surface barely in the depth of 3.8 and $1.2 \mu \mathrm{m}$ [11]. Similar small ranges of defects of order of $9.5 \mu \mathrm{m}$ are observed in GaAs wafers [12]. In this case, the vacancy clusters containing at least two vacancies were identified. These studies were performed using a small accelerator which allows implanting positrons at a definite depth of a few micrometers and scanning the regions close to the surface.

In this article, we intend to compare the SZ created in a stainless steel (SS) during cutting using three different methods, i.e., the traditional milling cutting (MC), laser cutting (LC), and abrasive water jet (AWJ). The positron annihilation methods, i.e., the conventional measurements of the positron lifetime and Doppler broadening of the annihilation line, were used for determining of properties of the SZ and its total range. In addition, the surface characteristics obtained using Wyko ${ }^{\circledR}$ Optical Profilers are included.

\section{Experimental Details}

\subsection{Sample Preparation}

All the studied samples of 304 type SS $(0.06 \% \mathrm{C}, 1.0 \% \mathrm{Si}$, $2.0 \% \mathrm{Mn}, 0.043 \% \mathrm{P}, 0.015 \mathrm{~S}, 0.1 \mathrm{~N}, 17 \% \mathrm{Cr}$, and $10 \% \mathrm{Ni})$ were prepared in the shape of small blocks with the dimensions of $5 \mathrm{~mm} \times 5 \mathrm{~mm} \times 10 \mathrm{~mm}$. To ensure the identical initial properties, the samples were annealed before cutting. The samples were annealed under vacuum conditions at $700{ }^{\circ} \mathrm{C}$ for $1 \mathrm{~h}$, and then slowly cooled to the room temperature. This procedure allowed us to remove stress and other defects induced during preparation, which could disturb the studied processes. Then, the samples were cut into two pieces, which were used for measurements.

The LC is the process where the energy of laser beam (continuous or pulse) causes smelting or smelting and evaporation of the cutting material. The density of laser beam power has to be between $10^{4}$ and $10^{8} \mathrm{~W} / \mathrm{mm}^{2}$. It determines a narrow zone of heat influence. The cutting was performed with a Trumpf 4003 from the ABC Colorex Group in Cracow (Poland). The 10-mm-thick sample was cut with the power of laser beam of 3,200 W. The nozzle moved at a speed of $0.2 \mathrm{~m} / \mathrm{min}$, and the focal length of the lens was set on 7.5 Zoll. The width of cutting slit was $0.2 \mathrm{~mm}$. The melted-through material was removed by air under the pressure of 20 bar.

In AWJ machining, the material removal occurs through erosion and results from interaction between an abrasive laden water jet and a specimen. The water jet pressure is even $400 \mathrm{MPa}$, and velocity of particles of water is three times as much as the speed of sound. It enables to provide the precise cutting of very complicated shapes with high quality of cutting edges. This machining was performed in another commercial company with water jet cutter working on high pressure pumps KMT Water jet System STREAMLINE $^{\mathrm{TM}}$. The samples were cut at a speed of $53 \mathrm{~mm} / \mathrm{min}$ under the jet pressure of about $34 \mathrm{MPa}$. In this case, the water flux was enriched by abrasive substance in the form of powder of garnet (hardness 7.5-8 Mohs, 80 mesh). The temperature of edges was about $40{ }^{\circ} \mathrm{C}$.

In the traditional MC, the turner knife removes pieces of the specimen step by step. The material moves fast in the front of the knife. In comparison to the cutting techniques mentioned above, significant material losses accompanied this process.

\subsection{The Positron Lifetime and Doppler Broadening Measurements}

For the positron lifetime measurement, fast-fast positron lifetime spectrometer with $\mathrm{BaF}_{2}$ scintillators was used. The time resolution (FWHM) of the lifetime spectrometer was equal to $260 \mathrm{ps}$. The positron source, i.e., ${ }^{22} \mathrm{Na}$ enveloped in the 7- $\mu \mathrm{m}$ kapton foil was located between the surfaces of the two identical pieces of samples, and this sandwich was positioned in front of the scintillator detectors of the positron lifetime spectrometer. The positron lifetime spectrum was measured during $24 \mathrm{~h}$ to obtain more than $2 \times 10^{6}$ counts in the spectrum. The average implantation depth, which is the reciprocal value of the linear absorption coefficient [13], for positrons emitted from ${ }^{22} \mathrm{Na}$ in steel, is ca. $29 \mu \mathrm{m}$. This value indicates the depth from the measured surface which is scanned by positrons in each measurement. About $63 \%$ of emitted positrons gets annihilated in the layer of such a thickness.

We noticed that, in our former studies, the measurements of the Doppler broadening of annihilation line gives more accurate results on the defect profile in steel and iron than the measurement of the positron lifetime. Partially it is because the positron lifetime spectrum is a raw result which must be deconvoluted using fitting procedure with several parameters. Measurements of Doppler broadening of the annihilation line were performed using a $\mathrm{HpGe}$ detector. The energy resolution of the HpGe detector system was equal to $1.38 \mathrm{keV}$ at $511 \mathrm{keV}$. As in the case of positron lifetime measurements, the positron source was sandwiched between two samples. For Doppler broadening measurements, the ${ }^{68} \mathrm{Ge} /{ }^{68} \mathrm{Ga}$ positron emitter for which the end point energy is $1.88 \mathrm{MeV}$ was applied, and this indicates the average positron implantation range in the studied steel equal to $159 \mu \mathrm{m}$. The ${ }^{68} \mathrm{Ge} /{ }^{68} \mathrm{Ga}$ emitter is a convenient source, because the peak-to-background ratio for annihilation line is better by factor of 4 than that for ${ }^{22} \mathrm{Na}$ emitter. According to our considerations, see [14], the 
larger implantation depth for the ${ }^{68} \mathrm{Ge} /{ }^{68} \mathrm{Ga}$ positrons does not affect the obtained defect profile if it smoothly decays with the depth increase.

\subsection{The Surface Characterization Equipment}

The surface of the cut samples were studied by Optical Profiler Veeco ${ }^{\circledR}$ Wyko NT 9300. The 2D and 3D analysis were performed using magnifications of $\times 2.7, \times 5.0, \times 10.0$, $\times 20.1$ and $\times 40.2$.

\section{Results and Discussion}

\subsection{The Surface Characterization}

The optical images of the cut surface are presented in Fig. 1. It can be seen that the surface topography differs for different cutting methods. The numerous regular scratches induced by a knife are visible at the surface machined using MC, Fig. 1a. AWJ machining induced a uniform surface with small irregularities dictated primarily by abrasive particles in the upper part of the cut; however, in the lower part of the cut, a wavy surface texture is recognized, Fig. 1b. The surface produced by LC exhibits deeper irregularities which are arranged in zones, Fig. 1c. More details can be found in the profiler images of the cut surfaces, Fig. 2.

Profiler images for MC indicate two families of the lays with different inclination in relation to the specimen edges (Fig. 2a). Cut pattern produced by LC (Fig. 2c) is much more subtle than that produced by $\mathrm{MC}$, and additionally, it is much more homogenous than that produced by AWJ (Fig. 2a, b). Profiler images obtained at various magnifications indicate one family of fine striation in direction roughly parallel to direction of the laser beam (Fig. 2c). On the other hand, three zones with different cut pattern (referred to as the ruff wave pattern) can be seen in the case of AWJ-machined specimen (Fig. 2b). One family of ruff waves with the direction close to the direction of the water jet is a characteristic feature of the cut-created surface. The cut surfaces were characterized by the roughness parameters. Two parameters have been chosen. There are the height 3D areal parameters $S_{\mathrm{a}}$ and $S_{\mathrm{ku}}$ defined in the standard ISO 25178 and clearly described by Bhushan [15]. The $S_{\text {a }}$ parameter is the arithmetic mean of the absolute values of the surface departures from the mean plane. This parameter is helpful for both detecting variations in the height of the surface and controlling a current manufacturing process. Any changes in the $S_{\text {a }}$ parameter indicate changes in the process. Kurtosis, i.e., the $S_{\mathrm{ku}}$ parameter allows us to assess machined surfaces, and sometimes, it is used to control stress fracture. It is applied for measuring spikiness of the surface and randomness of surface heights. The value of $S_{\mathrm{ku}}$ depends on the quality of the surface. For spiky surfaces, the $S_{\mathrm{ku}}$ parameter is bigger than 3, for bumpy ones it is smaller than 3 , and for the random surfaces it equals to 3 . The $S_{\mathrm{ku}}$ parameter is high in the case of a high proportion of the surface falling within a narrow range of heights. The farther the value is from 3 , the less repetitive the surface is. The values of the $S_{\mathrm{a}}$ and $S_{\mathrm{ku}}$ parameters measured for the surfaces of the three samples are collected in Table 1.

The $S_{\text {a }}$ parameter, i.e. the average roughness, for MC equals $1.1 \mu \mathrm{m}$ and is the smallest one in comparison to other cutting methods. The roughness of surfaces created by AWJ and LC is five times higher and is similar for both machining. Cutting with these techniques leaves a little number of deformations but with bigger height. This feature is also visible in Fig. 2b, c. The $S_{\text {ku }}$ parameter for all cases exceeds the value 3 . In addition, if we take deviations into account, these values are oscillating around 3.5. It points out that studied surfaces contain the spiky deformations.

The cutting technique has a significant influence on the quality of created surface. It is very clearly visible even with naked eye. One can expect the great impact on the structure of the SZ as well.
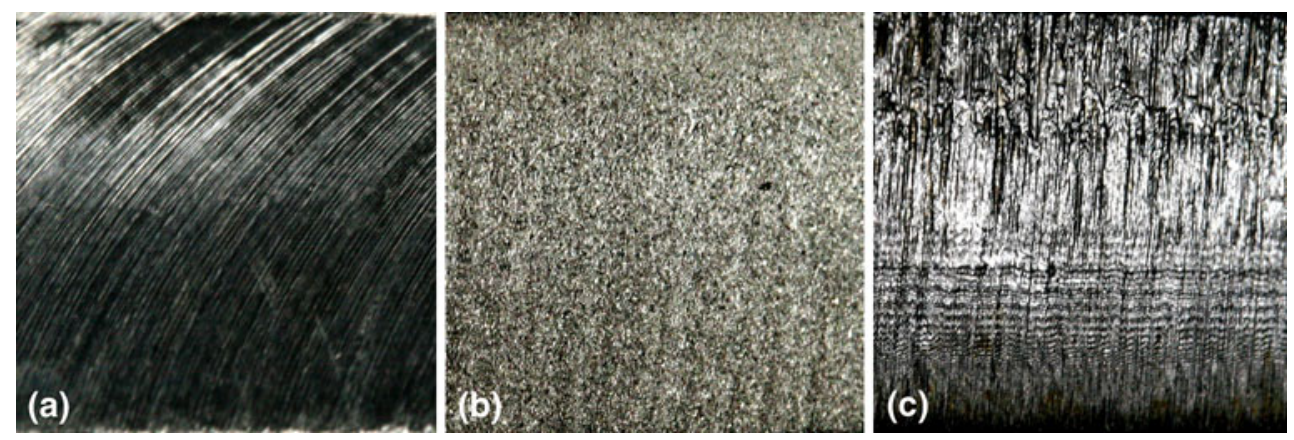

Fig. 1 The optical images of the cut surfaces of the SS samples machined by three different techniques. On the left the cut surface obtained using MC (a), the AWJ (b), and the laser beam LC (c) 

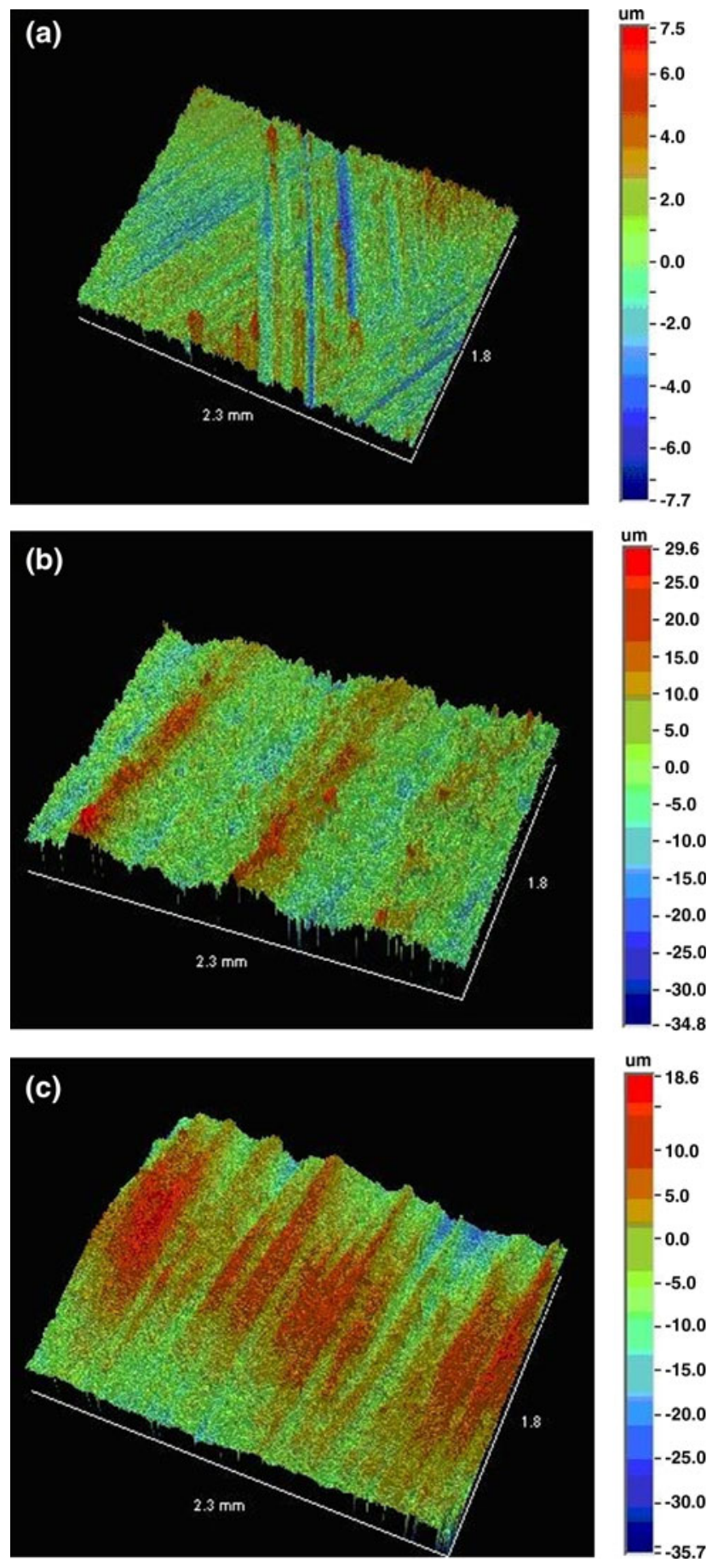

Fig. 2 Optical profiler images of the cut surface of the SS sample created by the MC (a), AWJ (b), and LC (c). Magnification is equal to 2.7

\subsection{Positron Lifetime Measurements}

Before the measurements of the defect profile, the positron lifetime spectrum was measured on the surfaces created by cutting. Diameter of the spot of the positron ${ }^{22} \mathrm{Na}$ source is about $3 \mathrm{~mm}$. Thus, the region of this diameter and the
Table 1 The average values of $S_{\mathrm{a}}, S_{\mathrm{ku}}$ parameters which characterize the roughness of the surface created by cutting

\begin{tabular}{lll}
\hline Cutting process & $S_{\mathrm{a}}(\mu \mathrm{m})$ & $S_{\mathrm{ku}}$ \\
\hline LC & $5.87 \pm 1.28$ & $3.02 \pm 0.43$ \\
AWJ & $5.38 \pm 0.72$ & $3.31 \pm 0.18$ \\
MC & $1.10 \pm 0.13$ & $3.99 \pm 0.55$ \\
\hline
\end{tabular}

For the description of the parameters see text

Table 2 The values of the positron lifetime measured directly on the cut surface for different cutting techniques

\begin{tabular}{ll}
\hline Cutting process & Positron lifetime \\
\hline Bulk value & $109.6 \mathrm{ps}$ \\
LC & $122.6 \mathrm{ps}$ intensity $78 \%$ \\
& $152.0 \mathrm{ps}$ intensity $22 \%$ \\
AWJ & $156.3 \mathrm{ps}$ \\
MC & $166.6 \mathrm{ps}$ \\
\hline
\end{tabular}

The positron lifetime accuracy is $\pm 1 \mathrm{ps}$

depth of about $29 \mu \mathrm{m}$ at the central part on the cut surface was inspected. In the Table 2, we gathered the results of the obtained lifetime spectra deconvolution. For the wellannealed SS sample, the obtained value equals to $109.6 \pm 1 \mathrm{ps}$ and it represents the bulk value which corresponds well to the positron bulk lifetime in well-annealed pure iron [16]. For the sample whose surface was cut with LC technique the positron lifetime spectrum was decomposed into two lifetime components giving the $\chi^{2}$ close to the unity. The first component equal to $122 \pm 1$ ps indicates the presence of edge dislocations. Certain amount of vacancies on the edge dislocations is pointed out by the value of the second lifetime component equal to $152 \pm 1$ ps [17]. The mean positron lifetime is about $129 \pm 1 \mathrm{ps}$ and it is slightly higher than the bulk value. Positron lifetime spectrum measured for AWJ cutting sample exhibits only single lifetime component equals to $156.3 \pm 1 \mathrm{ps}$ [16]. This value is attributed to the saturation of the positron trapping at the vacancies on edge dislocations. It is reported that highly deformed iron samples with the tensile strain of about $50 \%$ exhibits the single lifetime component in the measured spectrum above $160 \mathrm{ps}$ [18]. Similar value, i.e., $166.6 \pm 1 \mathrm{ps}$ is detected in the sample which was cut using MC technique. (It should be noted that another sample cut with a hand saw also exhibits the single lifetime component in the measured positron lifetime spectrum, equal to $164 \pm 1$ ps.) The values gathered in the Table 2 indicate that laser beam modifies the cut surface region in lower degree in comparison to MC and AWJ. 
3.3 The Depth Profile of the SZ Detected by the Positron Measurements

For inspection of changes induced by machining below the surface of the samples, the procedure of sequenced etching and measurement of the positron characteristics was performed. For this purpose, we chose the S-parameter which is extracted from the energy spectrum of annihilation line measured using the $\mathrm{HpGe}$ detector. The so-called $\mathrm{S}$-parameter is closely connected to concentration of defects and the positron trapping efficiency which is a minor factor in our studies [12]. This parameter is defined as the integral of the central part of the measured $511 \mathrm{keV}$ line normalized to the total area under the annihilation line. It is a suitable line shape parameter that characterizes the line broadening. It is a good dynamic parameter for monitoring changes of concentration of open volume defects, like vacancies, vacancy clusters, and dislocation in crystalline structure of the studied material. Another parameter, i.e., the $\mathrm{W}$-parameter given by the integral of the wing part of the $511 \mathrm{keV}$ line gives information on the chemical environment of defects. Both parameters are commonly used for studies on defects in metals and alloys.

After the measurement of the $\mathrm{S}$ and $\mathrm{W}$ parameters, the samples were etched in hydrofluoric acid to reduce their total thickness, and the next measurement was performed. This sequential procedure allowed us to determine the depth profile in respect of these parameters that depend on the etched thickness, which is the depth from the machined surface.

Fig. 3 depicts the measured depth profiles of the $\mathrm{S}$-parameter below the cut surface for all the three samples machined with MC, AWJ, and LC. The hatched area represents the value of the S-parameter measured for the sample which contains only residual defects. It determines the bulk level for non-defective crystalline host. We expect that the $\mathrm{S}$-parameter reaches this value at the depth where the surface treatment does not influence the material.

The open circles in Fig. 3 correspond to the values of the S-parameter measured for MC sample and gray circles to AWJ-machining sample. In the both cases, the value of the S-parameter, and hence the concentration of defects, i.e., dislocation decorated by vacancies and jogs, decreases with the depth. The defect's concentration on the surface is definitely higher for $\mathrm{MC}$ in comparison to AWJ cutting. The depth, where the S-parameter reaches the bulk level determining the total range of the SZ, is about $150 \mu \mathrm{m}$ for MC, Fig. 3. It is three times larger than that created by AWJ which is only $40 \mu \mathrm{m}$. One should notice that the S-parameter profile in both cases exhibits almost liner dependency on the depth. This is different from that in pure copper cut using a lathe. In the latter case, the obtained profile exhibited exponential decrease with the depth increase [10]. It seems that contact of the knife in MC or the abrasive particles in AWJ with the sample is a source creating certain amount of dislocations. The SZ is created during the motion of dislocations from the damaged surface into the interior of the samples. Dislocations can stop at other dislocations or lattice imperfection, and finally the $\mathrm{SZ}$ is constituted. For the pure well-annealed metal, their motion is not disturbed so effectively by the impurities or other lattice imperfection as it is done in alloys. For this case, one can expect the other type of dependency.

In contrast to our result, there was no report evidencing hardening or subsurface deformation below the surface of the SS (ASI 304) AWJ-machined samples in the smooth cutting region of the kerf $[19,20]$. The authors used the Vickers microhardness for detection of the changes below the cut surface. We argue that positron annihilation methods are much more sensitive than traditional microhardness
Fig. 3 Dependency of the S-parameter on the depth below the cut surface for the SS samples machined by three different techniques: MC, LC, and AWJ. The hatched region represents the bulk value of the S-parameter obtained for the well-annealed SS sample. On the right, the S-parameter versus the $\mathrm{W}$-parameter values from the Doppler broadening spectra are depicted (a)

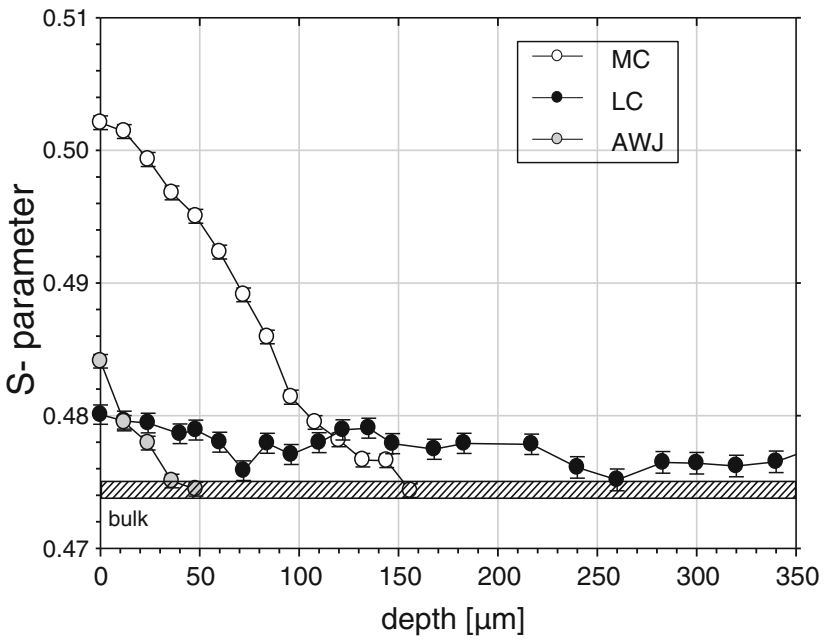

(b)

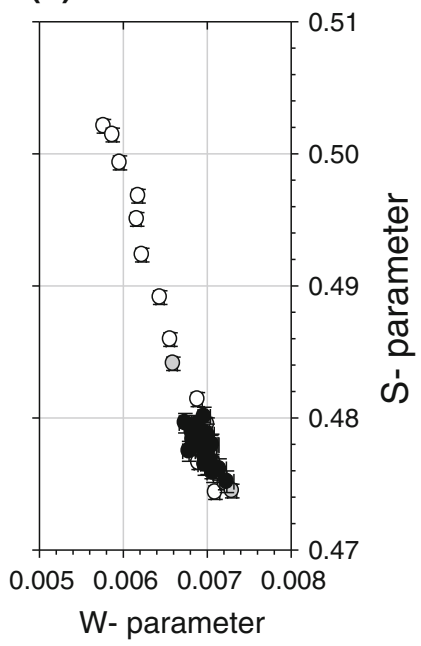


test. Certainly, one can expect that machining parameters can affect this depth and depth dependency of the S-parameter, but it requires future studies.

The well-defined SZ is detected also for LC machining (black points in Fig. 3); however, the depth profile of the $\mathrm{S}$-parameter differs significantly from that detected for MC and AWJ machining. The S-parameter is only slightly higher than the bulk value and remains almost constant up to the depth of about $500 \mu \mathrm{m}$. However, weak gradient of the S-parameter is observed. We argue that high temperature at the cut surface combined with fast cooling constitute the SZ with low but almost constant concentration of defects at large distance. Certainly, the motion of dislocation from the cut surface does not take place when the sample melts and evaporates.

Figure 3 (the right part) presents the $\mathrm{W}$-parameter versus the S-parameter plot for studied samples. All experimental points lie along the straight line. This correlation shows domination of the same kind of positron traps in the studied samples. No decoration of the traps by solute atoms takes place during the machining.

The tree cutting techniques affect the SZ below the cut surface in different way. It is clear that LC produces the minimal number of defects. They induce mainly during the fast cooling of the hot cut surface. MC creates the most damaged SZ with the linear gradient of defect concentration. This must be accompanied by increase of hardness in the SZ and can affect the functional properties of the material. However, this technique gives smoother surface than other machining techniques studied. AWJ produces fewer defects than MC which are distributed at small distance from the cut surface. The brief comparison of other engineering aspects of $\mathrm{LC}, \mathrm{AWJ}$, and $\mathrm{MC}$ machining was done by Betts in [21].

\section{Conclusions}

The studied machining, i.e., LC, AWJ, and MC methods influence both the cut surface and in the SZ. The surfaces created in these processes included a numerous deformation. MC enables us to obtain the smooth surface roughness of which in our case was five times smaller than roughness of LC and AWJ created surfaces. However, this treatment induces the SZ which is expanded to the depth of $150 \mu \mathrm{m}$ with additional high-defect concentration at the surface. $\mathrm{AWJ}$ also creates the $\mathrm{SZ}$, but its range is much smaller, i.e., $40 \mu \mathrm{m}$, with lower defect concentration at the surface. For LC, the SZ is marginally damaged up to the depth of 500 $\mu \mathrm{m}$. The positrons lifetimes measured on the cut surfaces point out the presence of edge dislocations and vacancies on dislocations. The surface roughness differs for these three machining techniques; however, it seems that it does not affect the SZ. Summarizing, we can state that MC induces the most damaged SZ, and LC machining induces the less damaged SZ.

Acknowledgments The authors express their gratitude to the Committee of Scientific Research (Poland), which supported this study under grant No. N N507 478637; to Tomasz Mularz and Mateusz Warot from the ABC Colorex Group in Cracow for devoting their time and help in preparing of the samples; and to Jarosław Dybowski for fruitful discussion.

Open Access This article is distributed under the terms of the Creative Commons Attribution Noncommercial License which permits any noncommercial use, distribution, and reproduction in any medium, provided the original author(s) and source are credited.

\section{References}

1. Rice, S., Nowotny, H., Wayle, S.: A survey of the development of subsurface zones in the wear of materials. Key Eng. Mater. 33, 77-100 (1989)

2. De Vries, M., Mastenbroek, A.: SEM observations of dislocation substructures around fatigue cracks in AISI type 304 stainless steel. Met. Trans. A 8, 1497-1499 (1977)

3. Zandrahimi, M., Reza Bateni, M., Poladi, A., Szpunar, J.: The formation of martensite during wear of AISI 304 stainless steel. Wear 263, 674-678 (1997)

4. Zhang, Y., Liu, J., Wang, J., Zeng, Y.: X-ray diffraction characteristics of five materials for stress measurement. J. Strain Anal. 45, 319-328 (2010)

5. Dryzek, J., Wesseling, C., Dryzek, E., Cleff, B.: Migration of vacancies in stainless steel measured by positron annihilation. Mater. Lett. 21, 209-214 (1994)

6. De Baerdemaeker, J., Colaux, J., Terwagne, G., Dauwe, C.: Investigation of nitrided 304L stainless-steel coatings by positron annihilation spectroscopy. Radiat. Phys. Chem. 68, 605-608 (2003)

7. Kim, J., Byrne, J.: Positron annihilation study of microvoids in centrifugally atomized 304 stainless steel. J. Appl. Phys. 73, 2489-2496 (2009)

8. Aly, E., Mohsen, M., Ahmed, E.: Investigation of precipitation in aluminum-copper alloy using positron annihilation lifetime technique. Egypt. J. Solids 31, 181-189 (2008)

9. Hansen, H., Petersen, K.: Surface damage from diamond-saw cutting of high purity mo studied by positrons. Phys. Status Solidi 69, 625-629 (1982)

10. Dryzek, J., Dryzek, E., Stegemann, T., Cleff, B.: Positron annihilation studies of subsurface zones in copper. Tribol. Lett. 3, 269-275 (1997)

11. McNeil, S., Lynn, K., Weber, M., Szeles, C., Soundararajan, R.: Positron-defect profiling in $\mathrm{Cd}_{1-\mathrm{x}} \mathrm{Zn}_{\mathrm{x}} \mathrm{Te}$ wafers after saw cutting. J. Electr. Mater. 29, 583-585 (2003)

12. Börner, F., Eichler, S., Polity, A., Krause-Rehberg, R.: Determination of the defect depth profile after saw cutting of GaAs wafers measured by positron annihilation. J. Appl. Phys. 84, 2255-2262 (1998)

13. Horodek, P., Dryzek, J.: GEANT4 simulation of implantation profiles for positrons injected in solids from radioactive sources ${ }^{22} \mathrm{Na}$ and ${ }^{68} \mathrm{Ge} /{ }^{68} \mathrm{Ga}$. Nukleonika 55, 17-19 (2010)

14. Dryzek, J.: Remarks on positron annihilation characteristics in nonhomogeneous systems. Nukleonika 42, 95-102 (1997)

15. Bhushan, B.: Introduction to Tribology. Wiley, New York (2002) 
16. Campillo Robles, J., Ogando, E., Plazaola, F.: Positron lifetime calculation for the elements of the periodic table. J. Phys. Condens. Matter 19, 176222-176241 (2007)

17. Hori, K., Koike, K., Oshima, R.: Investigation of lattice defects in the early stage of fatigue in iron by positron annihilation techniques. Appl. Surf. Sci. 242, 304-312 (2005)

18. Somieski, B., Krause-Rehberg, R.: Application of the positron lifetime spectroscopy as a method of non-destructive testing. Mater. Sci. Forum 175-178, 989-992 (1995)
19. Arola, D., Ramulu, M.: Material removal in abrasive water jet machining of metals Surface integrity and texture. Wear 210, 50-58 (1997)

20. Hashish, M.: Characteristics of surface machined with abrasive water jets. J. Eng. Mater. Technol. 113, 354-362 (1991)

21. Betts, B.: The kindest cut [laser and water cutting]. Eng. Technol. 5, 57-59 (2010) 